\title{
Determinantes y diferencias territoriales en materia de satisfacción laboral: el caso de Chile
}

\author{
Luz María Ferrada
}

\section{Resumen}

Este artículo analiza, mediante modelos logit binario y multinomial, el impacto de las condiciones objetivas de empleo y aspectos de percepción en la satisfacción laboral de los trabajadores. Además, se indaga en posibles diferencias entre la Región Metropolitana y otras zonas de Chile. Los datos provienen de la primera Encuesta Nacional de Condiciones de Empleo, Trabajo, Salud y Calidad de Vida de los Trabajadores y Trabajadoras en Chile (ENETS). Se concluye que el salario ejerce un impacto positivo sistemáticamente. Además, se estima que residir en una zona distinta de la Región Metropolitana tiene un efecto positivo y significativo; para verificarlo se aplica la técnica de emparejamiento, que confirma los resultados anteriores. Se concluye que distintos aspectos de apreciación subjetiva son altamente explicativos y su impacto es mayor que el de condiciones objetivas, lo que puede ser interesante tanto para la política pública como para la gestión de empresas.

\footnotetext{
Palabras clave

Empleo, condiciones de trabajo, salarios, satisfacción en el trabajo, medición, encuestas, modelos econométricos, Chile

Clasificación JEL

J280, M540, R230

Autora

Luz María Ferrada es Académica del Departamento de Ciencias Administrativas y Económicas de la Universidad de Los Lagos, Chile. Correo electrónico: Iferrada@ ulagos.cl.
} 



\section{Introducción}

El incremento sostenido que ha registrado el producto interno bruto (PIB) de Chile desde los años noventa ha estado asociado al aumento de la demanda laboral y de las remuneraciones en términos reales. Sobre la base de antecedentes suministrados por el Instituto Nacional de Estadísticas, entre 1993 y 2009 se observa un aumento de 1.802 .064 ocupaciones (INE, s/f) y del 1\% en el promedio de las remuneraciones anuales sobre la variación del índice de precios al consumidor (IPC) (INE, s/f); sin embargo, según diversos estudios, se concluye que dichos resultados no han permitido reducir las disparidades de salario entre territorios y que la calidad del empleo es aún muy baja. En consecuencia, cabe preguntarse cuán satisfechos con su trabajo están los trabajadores en Chile, qué factores son los que influyen en dicha evaluación y si existen diferencias notables en los resultados entre territorios.

En la literatura internacional se constata que la percepción que tienen los trabajadores respecto de su labor impacta de forma significativa en diversos aspectos de la vida, lo que es quizás el argumento más poderoso para justificar la gran cantidad de estudios internacionales sobre esta materia. No obstante, su desarrollo en el campo de la economía es reciente, sobre todo en Chile; el mayor desarrollo de la investigación en esta temática se encuentra en el ámbito de la psicología, aunque también se observan trabajos ligados a la administración de recursos humanos, en particular para estudiar la situación de determinadas industrias. La aportación principal de esta investigación consiste precisamente en indagar en un campo que ha sido poco estudiado en Chile, más aún cuando en este caso se incorpora el territorio. En este contexto se plantea un enfoque de investigación novedoso no abordado antes en Chile para el objeto de estudio.

La satisfacción laboral se define de distintas formas, según las disciplinas; un enfoque genérico al que recurren muchas investigaciones es entenderla como un estado emocional producido por la percepción subjetiva que el sujeto tiene de sus experiencias laborales, estado emocional también descrito como "el grado de bienestar que experimenta el trabajador con motivo de su trabajo"1. También se indica que es un reflejo de la utilidad que le proporciona la ocupación al trabajador, es decir, cómo este satisface sus requerimientos o expectativas y aporta a su calidad de vida. Por lo tanto, entre sus determinantes se cuentan tanto aspectos objetivos como subjetivos.

Los objetivos específicos de esta investigación son: i) evaluar el impacto de variables asociadas a condiciones objetivas del empleo y aspectos de percepción respecto de la organización y el entorno sobre la satisfacción laboral, y ii) verificar diferencias en materia de satisfacción laboral entre la Región Metropolitana y las demás zonas de Chile.

El trabajo se desarrolla con información de la única Encuesta Nacional de Condiciones de Empleo, Trabajo, Salud y Calidad de Vida de los Trabajadores y Trabajadoras en Chile (ENETS), realizada entre los años 2009 y 2010, que tiene representación regional y ofrece información de aspectos tanto objetivos como subjetivos del trabajo, incluido un conjunto de preguntas relativas a la satisfacción laboral.

Este artículo tiene cinco secciones, incluida esta introducción. En la segunda sección se exponen los antecedentes bibliográficos, en la tercera sección se describe la metodología utilizada, mientras que en la cuarta sección se presentan los resultados del estudio, conforme a los objetivos específicos señalados. Finalmente, en la quinta sección se resumen las principales conclusiones del estudio.

\footnotetext{
1 Véase [en línea] http://www.insht.es/InshtWeb/Contenidos/Documentacion/TextosOnline/GuiasMonitor/Psicosociologia/l/ Ficheros/psi23.pdf.
} 


\section{Antecedentes bibliográficos}

\section{Enfoques}

La investigación sobre satisfacción laboral se ha abordado desde distintas disciplinas, como la psicología, la administración de recursos humanos y la economía; cada una utiliza enfoques y herramientas propias. Sin embargo, los estudios que indagan en el impacto que tienen determinados factores en la evaluación que los sujetos hacen de su satisfacción laboral en general provienen de la psicología; en este campo se usa con frecuencia un modelo denominado bifactorial, que distingue entre factores extrínsecos (o higiénicos) e intrínsecos (o motivacionales). Los primeros contribuyen disminuyendo la insatisfacción, en cambio los otros incrementan el nivel de satisfacción. A su vez, los extrínsecos se relacionan con el entorno de trabajo y los intrínsecos reflejan las experiencias de los individuos con este último (Bòria-Reverter, Crespi-Vallbona y Mascarilla-Miró, 2012); varios estudios económicos también han abordado el problema siguiendo esta clasificación.

La investigación económica sobre satisfacción laboral es reciente. Probablemente, su desarrollo se asocia a la evolución epistemológica del trabajo en esta disciplina. En efecto, a principios del siglo XX, debido a la fuerte influencia de la revolución industrial, para algunos investigadores el trabajo era concebido como la actividad que produce riqueza (enfoque de Adam Smith), mientras que para otros se visualiza como el tiempo que se traslada al producto (enfoque de Karl Marx) o bien como lo que confiere valor a las mercancías (enfoque de David Ricardo) (Olarte, 2011). Sin embargo, son los trabajos de la década de 1960 en adelante los que destacan el capital humano (Mincer, 1958; Becker, 1964) y los resultados de investigaciones en las otras disciplinas los que motivan el interés por el estudio de la satisfacción laboral. De esta forma, se plantea que puede ser un factor relevante para la productividad, el crecimiento y la estabilidad. Un hito importante aquí son las investigaciones a partir de Freeman (1977).

En esta lógica, la gestión del trabajo se torna un ámbito cada vez más fundamental en la administración de la empresa. Su evolución se ilustra en tres enfoques. En primer lugar, el de Taylor, que destaca modelos que mejoren el esfuerzo físico, individual y colectivo, para lograr una mayor productividad del trabajo (Chiavenato, 2006). Posteriormente, el estudio se centra en el análisis del comportamiento organizacional (Stephen y Timothy, 2013), que postula que a través de la motivación y la satisfacción laboral sería posible alcanzar los objetivos de la empresa (Herzberg, Mausner y Snyderman, 1959; Herzberg, 1962). Otro enfoque defiende la implementación en las organizaciones de la gestión del recurso humano, es decir, la nueva gestión, cuya tesis consiste en tener un estilo de gestión que permita lograr una relación entre empresarios y trabajadores sobre la base de una filosofía de la colaboración que lleve a que los trabajadores se responsabilicen e identifiquen con los objetivos, incorporando los valores de la empresa (Narbona, 2012). Así, se genera una relación subjetiva con el trabajo que va más allá del contrato laboral, que produciría felicidad. En este sentido, los autores también plantean un control sutil, que se favorece en condiciones de flexibilidad laboral y bajos niveles de sindicalización (Narbona, 2012).

\section{Sobre la medición de la satisfacción laboral}

Medir la satisfacción laboral constituye un desafío, puesto que involucra aspectos difíciles de evaluar. Al respecto, existen varios enfoques, el más frecuente consiste en considerar que la satisfacción laboral se puede estimar a través de la utilidad que le proporciona el trabajo al trabajador (Olarte, 2011) y, por lo tanto, una buena variable ficticia (proxy) es el salario que está dispuesto a aceptar. Desde esta perspectiva los salarios dependerán tanto de la dotación de capital humano como de las 
características del trabajo (más competencias, habilidades personales, dedicación, entre otras) y su entorno (movilidad en la empresa, sindicatos, redes, oportunidades laborales y otras).

Sin embargo, la utilidad que el puesto de trabajo le proporciona a un individuo se puede evaluar también a través de la calidad de su empleo y de su vida laboral. En este marco es frecuente en la literatura latinoamericana el enfoque de trabajo decente (OIT, 1999), en el cual se indica que un trabajo es de calidad en la medida en que cuente con condiciones de libertad, equidad, seguridad y dignidad humana, con derechos protegidos, con remuneración adecuada y protección social, y se estudia a partir de factores objetivos. Farné (2003) acota el concepto de condiciones laborales a cuatro dimensiones: ingreso, jornada laboral, seguridad social y contrato laboral. No obstante, hay otros enfoques, como el de expansión de capacidades (Sen, 2000), que plantea que los individuos cuentan con funcionalidades, es decir, un conjunto de características personales (sexo, edad, nivel educativo, entre otros) y laborales (tipo de contrato, jornada de trabajo, por ejemplo) asociadas a su empleo que se transforman en capacidades (capacidad de generar altos ingresos o de mantenerse ocupado, entre otras), las que el individuo valora pues le permiten alcanzar un mayor nivel de bienestar (Sehnbruch, 2008). Es decir, un sujeto puede tener un trabajo (el bien) pero va a ser de buena calidad cuando se pueda convertir en una funcionalidad que él valore; de esta forma las particularidades del trabajo combinadas con las necesidades y características de la persona determinan las capacidades que esa ocupación puede generarle (Sehnbruch, 2012). No obstante, otros investigadores han definido el concepto incluyendo de forma explícita aspectos subjetivos. Reinecke y Valenzuela (2000) lo describen como "el conjunto de factores vinculados al trabajo que influyen en el bienestar económico, social, psíquico y de salud de los trabajadores" (Farné, 2012).

En un tercer grupo de investigaciones se evalúa la satisfacción laboral mediante la percepción que muestran directamente los sujetos de su trabajo. En este caso, los trabajadores responden a preguntas a través de una escala que indica el grado de satisfacción o insatisfacción con el trabajo. Así pues, se han diseñado diversos cuestionarios y escalas de medición (Olarte, 2011).

Finalmente, hay varios estudios en que se estima el nivel de satisfacción relativa mediante un análisis multivariado, esto es, se construye un indicador sintético incorporando distintas dimensiones del concepto, ya sean objetivas o subjetivas (Somarriba y otros, 2010). Estos son los factores extrínsecos e intrínsecos del modelo bifactorial, como características del individuo, que la empresa no puede modificar pero que inciden en la satisfacción relativa que pueden tener distintos grupos, caracterizados por variables demográficas y de residencia, entre otras. Otro tipo de factores son aquellos aspiracionales, es decir, los que nunca se logran satisfacer por completo y que por lo tanto son motivadores permanentes (McGregor, 1981), como la autoestima, que estarían relacionados tanto por sus expectativas como por su historia de vida.

En este estudio se analiza la satisfacción laboral a partir de las percepciones que los trabajadores asalariados reportan en una escala de siete puntos, cuando responden a la pregunta ¿cómo se siente usted con su trabajo? (pregunta D1h, ENETS módulo D), para luego investigar en qué medida ciertos factores (objetivos y subjetivos) impactan en ella.

\section{Determinantes de la satisfacción laboral}

Uno de los aspectos más estudiados es la relación entre satisfacción y salario. Siguiendo el enfoque utilitarista el resultado esperado es una correlación positiva entre ambas variables, inclusive se espera una relación causal en que un mayor salario determine una mayor satisfacción laboral. Sin embargo, este resultado no siempre es tan evidente; por ejemplo, Clark y Oswald (1996) indican lo contrario; otros (Bòria-Reverter, Crespi-Vallbona y Mascarilla-Miró, 2012) plantean que algunos trabajadores podrían estar descontentos con su salario, pero estar satisfechos en general. Aun así, Borra y Gómez (2012) 
y Farné y Vergara (2007) constatan un impacto positivo. Posiblemente la respuesta depende de otras variables, como el estar en la parte alta o baja de la distribución de ingresos. Se indica que lo que suele ocurrir es que aquellos que optan por salarios inferiores revelan mayor satisfacción porque valoran el hecho de tener trabajo, por sobre sus características monetarias. Card y otros (2010) señalan que la respuesta también podría estar afectada por comparaciones de salarios relativos y que esta relación no es lineal, aun cuando Clark, Nicolai y Westergård-Nielsen (2007) indican que la comparación de salario podría ser positiva en la satisfacción laboral si el trabajador viese en ella una expectativa de ingreso futuro.

Igualmente, con otros factores objetivos se observan diversos resultados. En cuanto a las horas de trabajo y el tipo de contrato, Gamero (2003) obtiene que en general los trabajadores españoles, en particular los varones, no prefieren trabajos parciales, además verifica que hay indiferencia entre contrato privado o público, aunque en otros trabajos se indica una posición favorable en los empleos del sector público, como sostienen De Vries y otros (2015). Booth y Van Ours (2007), utilizando una encuesta de panel de hogares británicos, encuentran comportamientos distintos según el sexo, dado que mientras los hombres no mejoran su satisfacción con las horas de trabajo, las mujeres sí y prefieren horas de trabajo parcial.

En el mismo sentido, otros determinantes estudiados son la estabilidad en el puesto de trabajo (Clark y Postel-Vinay, 2009; Bòria-Reverter, Crespi-Vallbona y Mascarilla-Miró, 2012) o las posibilidades de ascenso; la literatura indica que los trabajadores se manifiestan más infelices cuando no pueden ascender, inclusive frente a un aumento de salario (Grund y Sliwka, 2007).

Como indican Bòria-Reverter, Crespi-Vallbona y Mascarilla-Miró (2012), en este campo no es suficiente considerar solo el salario o el puesto jerárquico sino también aspectos como las posibilidades de participación y el sentimiento de utilidad que en definitiva se derivan de aspectos intrínsecos. Además, contribuyen algunas características organizacionales, por ejemplo se estima una relación positiva entre la satisfacción laboral y un mejor clima organizacional (Juárez-Adauta, 2012), así como las relaciones interpersonales (Juárez-Adauta, 2012).

A su vez, se ha visto que la percepción del nivel de satisfacción o insatisfacción es una respuesta que podría estar afectada por características del sujeto, entre ellas, aspectos sociodemográficos, como la edad, el estado civil, el sexo y el nivel educativo. En relación con el sexo, en algunos estudios se señala que, contrariamente a lo esperado, las mujeres revelan mayor satisfacción laboral que los varones (Clark, 1997), aunque el diferencial desaparece en jóvenes con educación superior. No obstante, Sloane y Williams (2000) detectan que una mayor satisfacción en mujeres no se deriva de los atributos del trabajo, ni de recompensas monetarias, sino de una diferencia innata asociada al género. Otra explicación es que ellas podrían tener menores posibilidades de trabajar por lo que cuando lo logran están satisfechas.

En cuanto al nivel educativo, se señala que las personas con menor nivel de educación y experiencia tendrían menores expectativas de optar a otros empleos, por lo que el nivel de insatisfacción por el no logro es más bajo, lo que también sucede con los salarios (Belfield y Harris, 2002; LévyGarboua, Montmarquette y Simonnet, 2007).

Otra variable muy estudiada son los sindicatos. Un resultado interesante y contrario a lo esperado es que pertenecer a alguna organización impactaría en una menor satisfacción laboral (Freeman, 1977). Esta situación podría explicarse porque es posible que los sindicalizados posean mayores expectativas derivadas de la organización sindical o bien porque los que se han sindicalizado pueden tener un umbral mayor de insatisfacción pues precisamente pueden haberse asociado para mejorar dicha percepción. También se plantea que la sindicalización podría tener un efecto endógeno, pues podría explicar el nivel de satisfacción y a la vez ser explicada por este, es decir, un efecto simultáneo (Bryson, Cappellari y Lucifora, 2004). Otros indican que la afiliación al sindicato tiene 
carácter endógeno y reduce la satisfacción laboral, en cambio estar cubierto por la organización sindical es totalmente exógeno y tiene un efecto positivo sobre la satisfacción (Rodríguez-Gutiérrez y Prieto-Rodríguez, 2004).

En otro ámbito, es posible que el impacto de estos factores en la satisfacción varíe según el lugar de residencia, probablemente por diferencias en las condiciones de su entorno que impactan en su percepción. Pouliakas y Theodossiou (2010) estudian la satisfacción laboral entre países europeos y verifican que la relación entre satisfacción y salario es distinta según el lugar: en el sur los trabajadores que optan a salarios bajos están menos contentos que los que perciben una remuneración alta, pero no ocurre lo mismo en el norte de Europa, lo que se relacionaría con la calidad del empleo. En el caso de España, Iglesias, Llorente y Dueñas (2010) estudian la relación entre calidad del empleo y satisfacción laboral, y constatan una elevada calidad del empleo en Madrid, en dos períodos estudiados, aunque los trabajadores manifiestan menores niveles de satisfacción laboral.

A su vez, se ha estudiado que las metrópolis tienen cierta capacidad de encantar a los trabajadores pues ofrecen una serie de atractivos. La literatura sobre los atractivos urbanos es reciente y ha permitido, entre otras cosas, explicar diferencias entre territorios en cuanto a salarios (Macedo y Simões, 1998) y precio de la vivienda (Rocha y Magalhães, 2013). En Chile los atractivos se han utilizado como una variable que explica las migraciones a ciertas regiones (Aroca, Geoffrey y Paredes, 2001), en particular la Región Metropolitana; inclusive se hace una clasificación entre regiones atractivas para vivir y atractivas para trabajar (Aroca y Atienza, 2008) y se plantea la hipótesis de que la variable impactaría en diferencias regionales de participación laboral femenina (Ferrada y Zarzosa, 2010) y en la conmutación de larga distancia (Jamett y Paredes, 2013). Si bien en este caso no se profundiza en el tema, se menciona pues permitiría argumentar algunos resultados de esta investigación.

El interés aquí es advertir posibles diferencias territoriales en cuanto a satisfacción laboral en Chile. Se sospecha que pueden existir desigualdades entre la Región Metropolitana y otras zonas de Chile debido a grandes disparidades salariales a favor de la Región Metropolitana y a una fuerte heterogeneidad espacial en cuanto a su matriz productiva, dado que dicha región concentra la oferta de atractivos y de servicios, principalmente financieros y administrativos, y consumo interno dado su peso demográfico, mientras que el resto de las regiones tienen en común marcadas especializaciones basadas en recursos naturales, con alta dependencia externa.

\section{Aspectos metodológicos}

Conforme a lo señalado, en este caso se estudia la satisfacción laboral a través de información directa, es decir, de la respuesta que dan los sujetos respecto de su situación laboral. Se utilizan modelos causales para evaluar en qué medida la satisfacción laboral que reportan los trabajadores asalariados de empresas privadas de Chile está determinada por factores objetivos, percepción, residencia o aspectos demográficos. Además, mediante metodología de evaluación de impacto -técnica de emparejamiento exacto (coarsened exact matching) - se verifican posibles diferencias en materia de satisfacción entre los que residen en la Región Metropolitana y las otras zonas del país. Para el análisis descriptivo se trabaja con el software Programa de Estadísticas de Ciencias Sociales y las estimaciones econométricas con Stata.

\section{Datos}

Como se ha indicado, se utilizan los datos de la primera Encuesta Nacional de Condiciones de Empleo, Trabajo, Salud y Calidad de Vida de los Trabajadores y Trabajadoras en Chile (en adelante ENETS), 
realizada en el período 2009-2010. Se trata de la única encuesta de este tipo aplicada en Chile, aunque su segunda versión está en estudio (MINSAL, 2011).

El cuestionario se aplica en los hogares, dato importante pues no necesariamente corresponde a la zona en que trabaja el sujeto encuestado. Además, son informantes individuos mayores de 15 años que hayan trabajado durante los 12 meses anteriores a la fecha en que se realiza el empadronamiento muestral. El marco muestral se basa en el Censo Nacional de Población y Vivienda de 2002 y la muestra es de 9.503 observaciones.

El cuestionario contiene información de todas las regiones de Chile, tanto en zonas urbanas como rurales, excluidos aquellos lugares de difícil acceso geográfico ${ }^{2}$. El diseño muestral aplicado permite representación regional, de forma que utilizando factores de expansión, incluidos en la base de datos, se puede realizar adecuadamente el análisis regional. En resumen, con los factores se obtiene que dichas unidades muestrales representen a 7.939.170 trabajadores de Chile distribuidos en sus 15 regiones (MINSAL, 2011).

Del total de observaciones de la muestra, 5.802 corresponden a trabajadores del sector privado asalariados (excluidos los trabajadores del servicio doméstico), que representan a 4.532.274 personas. La distribución de la muestra por región, con y sin ponderar, se aprecia en el cuadro 1. Sin embargo, a este nivel de desagregación la cantidad de observaciones en algunos territorios se reduce mucho, por lo que para el análisis territorial en este trabajo se agrupan las regiones, conformando siete zonas, de acuerdo a criterios de cercanías geográficas y características productivas, como se indica a continuación.

\section{Cuadro 1}

Muestra por región y zona (MZ)

(En número de personas)

\begin{tabular}{|c|c|c|c|}
\hline Región & Muestra & Muestra ponderada & Zonas \\
\hline Arica & 256 & 42891 & \multirow{2}{*}{ MZ1 } \\
\hline Tarapacá & 216 & 74926 & \\
\hline Antofagasta & 320 & 164428 & \multirow{2}{*}{ MZ2 } \\
\hline Atacama & 333 & 72532 & \\
\hline Coquimbo & 290 & 184691 & \multirow{2}{*}{ MZ3 } \\
\hline Valparaíso & 594 & 445357 & \\
\hline Metropolitana & 1199 & 1973304 & MZ4 \\
\hline O'Higgins & 375 & 280690 & \multirow{3}{*}{ MZ5 } \\
\hline Maule & 318 & 231788 & \\
\hline Biobío & 532 & 498832 & \\
\hline Araucanía & 275 & 204647 & \multirow{3}{*}{ MZ6 } \\
\hline Los Ríos & 288 & 83464 & \\
\hline Los Lagos & 280 & 208466 & \\
\hline Aysén & 246 & 24704 & \multirow{2}{*}{ MZ7 } \\
\hline Magallanes & 280 & 41554 & \\
\hline Total & 5802 & 4532274 & \\
\hline
\end{tabular}

Fuente: Elaboración propia, sobre la base de datos de la Encuesta Nacional de Condiciones de Empleo, Trabajo, Salud y Calidad de Vida de los Trabajadores y Trabajadoras en Chile.

\footnotetext{
2 Localidades de difícil acceso geográfico tales como: Ollagüe, Isla de Pascua y Juan Fernández, Lago Verde, Guaitecas, Chile Chico, Villa O'Higgins, Tortel, Torres del Paine, Río Verde, Laguna Blanca, San Gregorio, Primavera, Timaukel, Cabo de Hornos y Antártica. Además, dada la coyuntura nacional, se excluyeron también las comunas de Chaitén, Futaleufú y Palena.
} 


\section{Variables dependientes}

El fenómeno que se pretende estudiar es la satisfacción con el trabajo, lo que se analiza a partir de la pregunta ¿cómo se siente con su trabajo? formulada en la ENETS. Los encuestados tienen siete alternativas posibles para responder: muy mal, mal, menos que regular, regular, más que regular, bien y muy bien. Responden a esta pregunta 5.782 personas, lo que representa a 4.512 .913 trabajadores del sector privado. Los resultados en la población objeto de estudio se observan en el gráfico 1.

\section{Gráfico 1}

Frecuencia de la respuesta a la pregunta ¿cómo se siente con su trabajo? (En porcentajes)

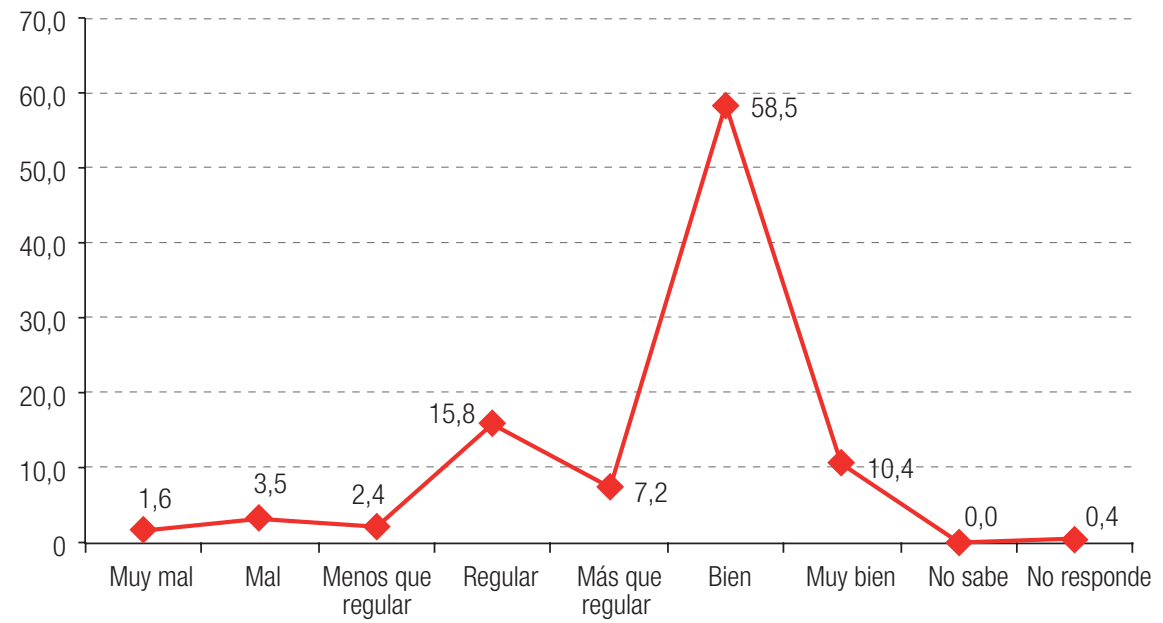

Fuente: Elaboración propia, sobre la base de datos de la Encuesta Nacional de Condiciones de Empleo, Trabajo, Salud y Calidad de Vida de los Trabajadores y Trabajadoras en Chile.

Como se aprecia, los niveles de satisfacción laboral declarados por los trabajadores son altos, lo que constituye otra motivación para indagar en los factores que impactan en esta percepción de los trabajadores entre territorios, dadas las conocidas y elevadas disparidades salariales en Chile y el alto nivel de concentración espacial.

\section{Metodología}

Considerando la distribución de las respuestas (véase el gráfico 1), el fenómeno se estudia de dos formas, mediante una variable binaria y otra categórica, de la siguiente forma:

- La variable binaria SATIS2 toma el valor 1 cuando los sujetos responden con las opciones muy bien o bien y 0 cuando responden con las otras opciones.

- La variable categórica SATIS3 se plantea en tres alternativas: valor 1 cuando responden muy mal, mal, menos que regular, regular o más que regular, 2 si responden bien y 3 en el caso de muy bien.

Para la primera situación se estima un modelo logit binario; como se verá más adelante, estas estimaciones incluyen efectos fijos para los territorios, los que resultan significativos. Para confirmar tales impactos se estiman las diferencias en materia de satisfacción laboral entre los que residen en la Región Metropolitana y el resto de las zonas del país utilizando un método de evaluación de impacto por emparejamiento exacto (Blackwell y otros, 2009). El otro modelo es un logit multinomial (Greene, 1999). 


\section{a) Modelo logit binario}

Como se ha señalado, en este caso la variable dependiente dicotómica es SATIS2. De esta forma, se evalúa cómo impactan ciertas variables en la probabilidad de que los trabajadores se sientan satisfechos o muy satisfechos. En este modelo los coeficientes se estiman por el método de máxima verosimilitud, que puede expresarse de la siguiente manera (Greene, 1999):

$$
\hat{P}(S A T I S 2=1)=\frac{e^{X, \hat{\beta}}}{1+e^{X, \hat{\beta}}}=\wedge(\beta, X)
$$

Donde $\hat{\beta}$ es el vector de los parámetros estimados y $X$ es la matriz de variables explicativas.

La interpretación se realiza sobre la base de los coeficientes estimados. Además, se calcula la razón de tasas frente a cada variable explicativa como:

$$
\Omega=\frac{P(S A T I S 2=1)}{P(S A T I S 2=0)}
$$

\section{b) Método de emparejamiento exacto}

Aun cuando en el logit anterior se incluye en las variables explicativas la zona de residencia de los trabajadores, con esta técnica es posible mejorar la comparación en materia de satisfacción laboral entre los que habitan en la Región Metropolitana y las otras zonas de Chile.

Si bien dicho método se usa sobre todo en la evaluación de políticas públicas, aquí se utiliza pues permite comparar individuos similares en cuanto a ciertas características. En este caso, se trata de los trabajadores que ofrecieron respuestas similares en las variables de la matriz $X$, definidas en la metodología anterior. Estas son las variables de control para el proceso de emparejamiento. Por lo tanto, en este ejercicio, la diferencia en materia de satisfacción laboral solo se explica por el hecho de ser un trabajador que reside en la Región Metropolitana o en otra zona del país.

Existen distintas técnicas de emparejamiento, en esta instancia se opta por el emparejamiento exacto dadas las ventajas indicadas en la literatura, que destaca que cumple adecuadamente con el principio de congruencia, restringe el emparejamiento de datos a las áreas que tienen un soporte común y es eficiente computacionalmente (Blackwell y otros, 2009).

El procedimiento consiste en realizar primero un emparejamiento y luego una estimación, en este caso un logit binario. El emparejamiento se realiza con las variables definidas en el logit binario.

Para ver si el proceso de emparejamiento es adecuado, con la técnica de emparejamiento exacto se calcula el estadístico $\$ 1$, que representa el desequilibrio entre los tratados y los no tratados, cuyo valor oscila entre 0 y 1 . La idea es que con el emparejamiento las distribuciones sean lo más iguales posibles, es decir, se debería obtener un $\$ 1$ cercano a 0 (lacus, King y Porro, 2012).

\section{c) Logit multinomial}

Con el objetivo de verificar cómo cambia el impacto de los determinantes cuando se analizan distintos niveles de satisfacción, se evalúan diferencias en los determinantes entre aquellos que están muy satisfechos, satisfechos y los otros, o sea, se agrupan las respuestas en tres categorías (SATIS3). En esta situación la variable dependiente tomará como referencia (categoría base) la primera 
alternativa (muy mal, mal, menos que regular, regular o más que regular), es decir, $\mathrm{J}=0$ constituye la categoría de referencia, lo que condiciona las interpretaciones de los coeficientes que se estiman para las otras opciones. De este modo, indicará el impacto en la probabilidad de que los trabajadores mejoren su nivel de satisfacción en el trabajo a satisfecho $(\mathrm{J}=2)$ o muy satisfecho $(\mathrm{J}=3)$. Esto es, para la referencia:

$$
\hat{P}(S A T I S 3=j)=\frac{e^{X, \hat{\beta}}}{1+\sum_{j=1}^{j=2} e^{X, \hat{\beta}}}
$$

Y la probabilidad de las otras posibilidades es:

$$
X \hat{\beta}=\alpha x+\rho x+\delta x
$$

Donde $\beta$ es el vector de coeficiente estimado y $X$ es la matriz de variables explicativas.

Los parámetros se estiman por máxima verosimilitud, luego, los estimadores serán aquellos que maximizan la probabilidad de la muestra observada. Para la interpretación del modelo se estima la razón de tasas, con lo cual se puede llegar a una lectura más clara de los modelos estimados (Long y Freese, 2014).

\section{Variables explicativas}

Conforme a las definiciones y evidencia empírica presentadas, se seleccionan variables para abordar cuatro tipos de características, luego:

$$
X \hat{\beta}=\alpha x+\varphi x+\rho x+\delta x
$$

donde $\alpha, \varphi, \rho$ y $\delta$ son los parámetros estimados que acompañan a las variables explicativas indicadas en cada caso, a saber, características demográficas, aspectos objetivos del empleo, zona de residencia y percepción del trabajador, respectivamente. Cada una de ellas se analiza conforme a las variables que se observan en el cuadro 2.

Inicialmente se analizó un conjunto más amplio de posibles determinantes, conforme a lo indagado en la literatura; sin embargo, antes de realizar estimaciones se estudiaron cada una de ellas aplicando un análisis descriptivo. Finalmente, se seleccionaron las variables del cuadro 2. Las otras se excluyeron ya sea porque no resultaron relevantes según el análisis previo, porque en algunas hay muchas respuestas omitidas o porque la cantidad de respuestas favorables es muy baja, como ocurre por ejemplo con la pregunta sobre afiliación sindical, o porque la cantidad de afiliados es muy baja, lo que anula la posibilidad de realizar algunos cruces cuando el análisis es por zona.

Del mismo análisis se obtienen las categorías de variables explicativas. Como se observa, resultan todas dicotómicas, dado que las respuestas a las variables de interés en la ENETS son en categorías. En este caso se indagaron distintas alternativas y por último se optó por las mencionadas en el cuadro 2. La única excepción que se indagó en forma continua fue escolaridad pero, conforme al análisis inicial, finalmente se descartó. 


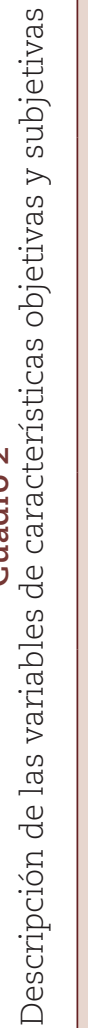

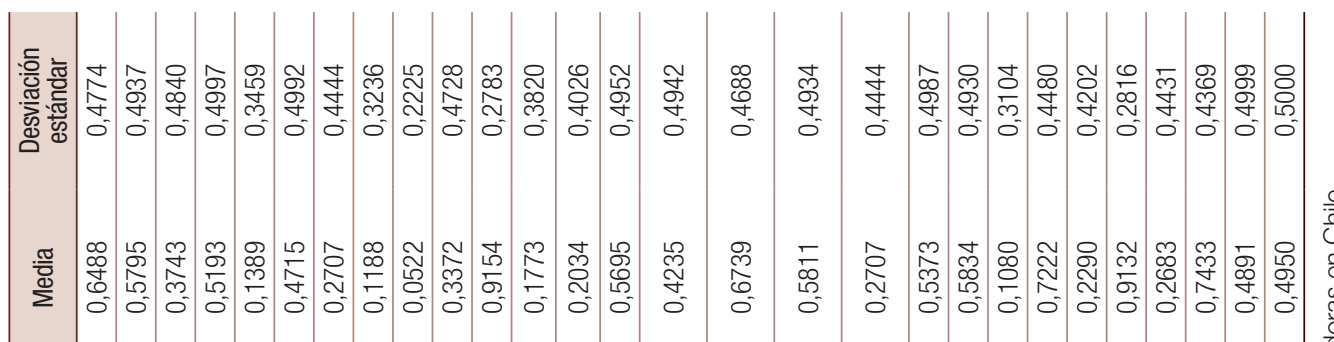

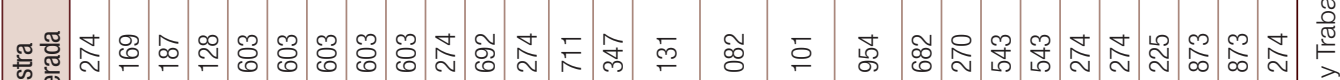
旁至

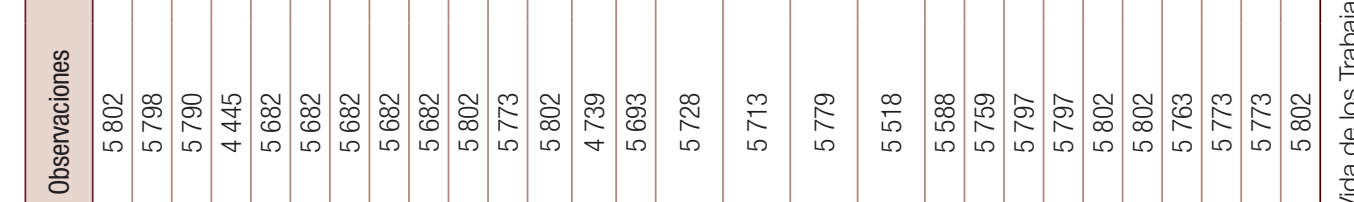

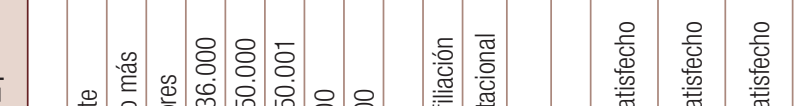

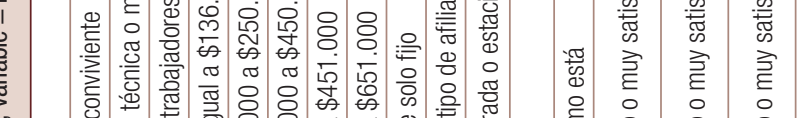

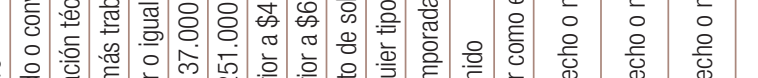

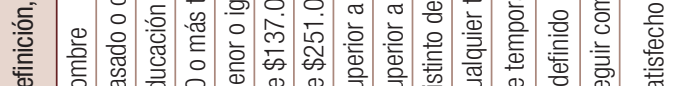

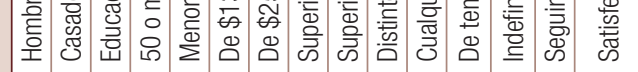

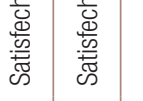

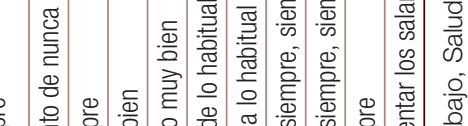

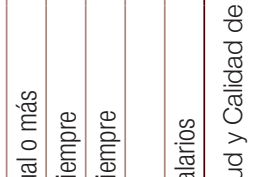

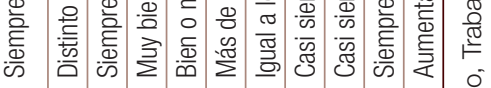

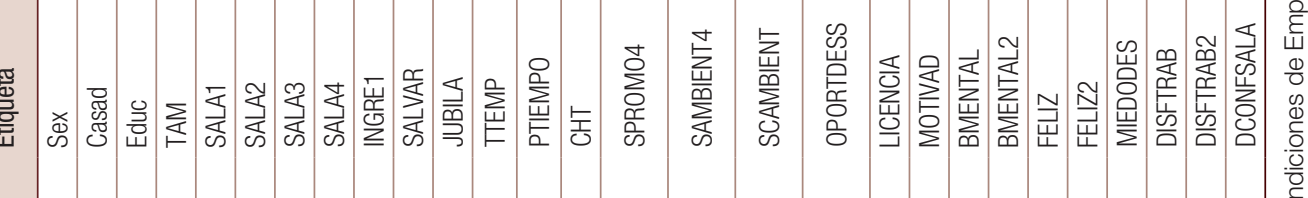

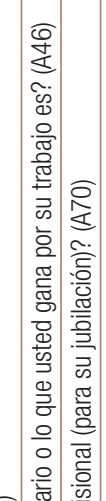

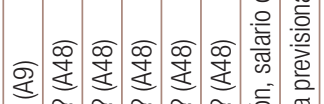

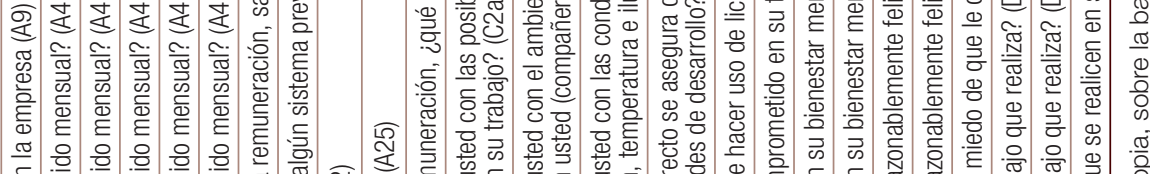

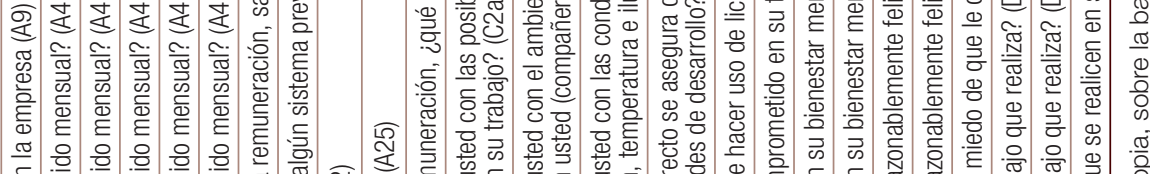

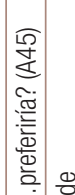

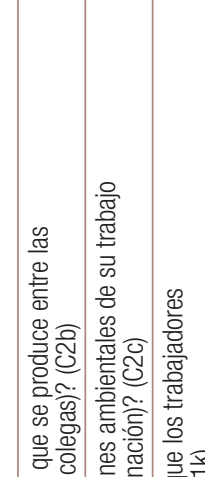

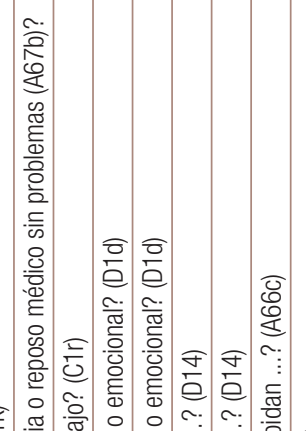

은

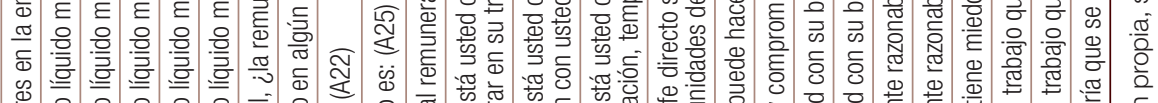

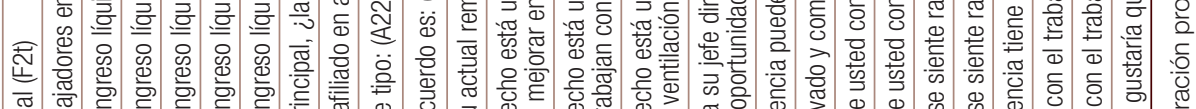

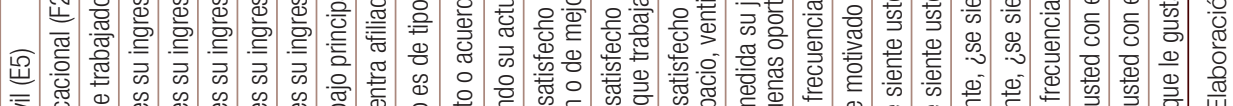

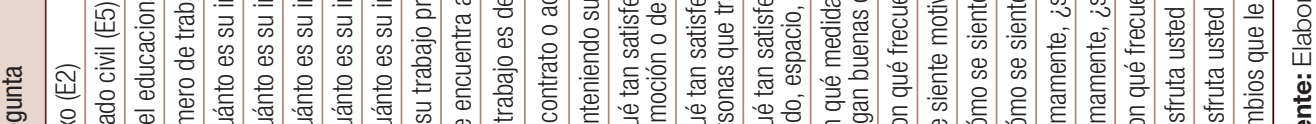

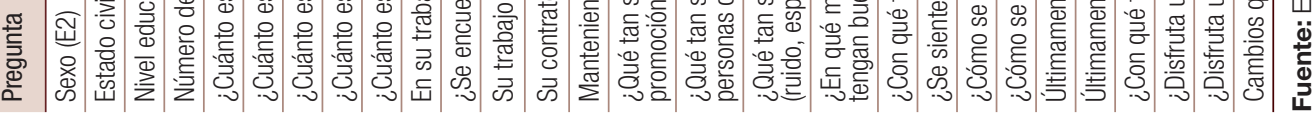




\section{Resultados}

En los cuadros 3, 4 y 5 se presentan los resultados de las regresiones logit, las estimaciones con y sin la aplicación del método de emparejamiento exacto y los resultados obtenidos con los modelos multinomiales, respectivamente. A continuación se describe el análisis para cada caso.

\section{Modelo logit}

En este caso se realizan tres estimaciones: en la primera se incluyen variables objetivas y de características del sujeto, en la siguiente se agregan las zonas y luego, variables de percepción.

Como se aprecia en el cuadro 3A, la probabilidad de que los trabajadores se sientan satisfechos o muy satisfechos aumenta significativamente en los varones, en los que tienen trabajo no temporal y en aquellos con mayor salario.

La mayoría de los trabajadores son hombres (65\%) y solo un tercio tiene un nivel educativo superior o al menos técnico proveniente de la enseñanza media técnica o comercial completa (véase el cuadro 2). En los primeros la propensión a estar satisfechos es un $28 \%$ mayor que en las mujeres; en cuanto al nivel educativo la variable no resulta significativa (véase el cuadro 3A).

La variable que más impacta es el salario de la ocupación principal. Alrededor de la mitad de los sujetos (47\%) indica que tiene un salario líquido mensual que oscila entre 137.000 pesos y 250.000 pesos (véase el cuadro 2). Al acceder a este tramo el nivel de satisfacción mejora significativamente, y en la medida en que se incrementa el tramo de ingreso el impacto es mayor (SALA3 y SALA4). A su vez, el tener contratos temporales o estacionales impacta negativamente.

Se debe considerar que, de acuerdo a la ENETS, un 13\% de los trabajadores asalariados del sector privado obtienen un ingreso mensual igual o inferior al salario mínimo legal y que un cuarto de ellos se ubica en valores levemente superiores a este. Además, la macrozona con mayor salario medio es la que corresponde a la Región Metropolitana (véase el gráfico 2)3.

Cabe mencionar que también se evalúan las variables estado civil, tamaño de la empresa (por el número de trabajadores), afiliación a sistemas de jubilación y si el salario es de tipo variable, las que no se incluyeron finalmente pues no son significativas ni aportan conjuntamente al modelo.

Sin embargo, cuando se incorporan las variables que se refieren a las macrozonas territoriales (véase el cuadro 3B), se obtiene siempre un coeficiente positivo y significativo, lo que apunta al hecho de que la residencia en una zona distinta de la Región Metropolitana (correspondiente a la macrozona de base en la estimación) impacta favorablemente en la satisfacción laboral y el efecto es menor en las macrozonas del norte de Chile. Este hallazgo es contrario a lo esperado: por un lado el salario afecta positivamente a la satisfacción, pero residir en zonas distintas de la de mayor salario (MZ4) impacta positivamente. Quizá en este resultado afecte el hecho de que, como se ha señalado, la encuesta se hace en el hogar y la respuesta corresponde a una persona de dicha familia, por lo tanto no necesariamente trabaja en la zona en que reside. Sobre este punto se profundizará en la sección siguiente.

En el cuadro 3C se presentan los resultados obtenidos con el tercer modelo, en que se incluyen variables objetivas y subjetivas. Como se aprecia, el salario sigue siendo significativo, no así la variable que indica temporalidad en el contrato.

\footnotetext{
3 En la Encuesta se consulta por valores líquidos. El salario mínimo en Chile en el año 2009 era 165.000 pesos y en 2010 172.000 pesos, ambos expresados en valores brutos mensuales, lo que representa aproximadamente 140.000 pesos y 146.000 pesos líquidos, es decir, una vez descontados los seguros y la seguridad social.
} 


\section{Gráfico 2}

Salarios líquidos declarados, por macrozonas

(En pesos chilenos y porcentajes)

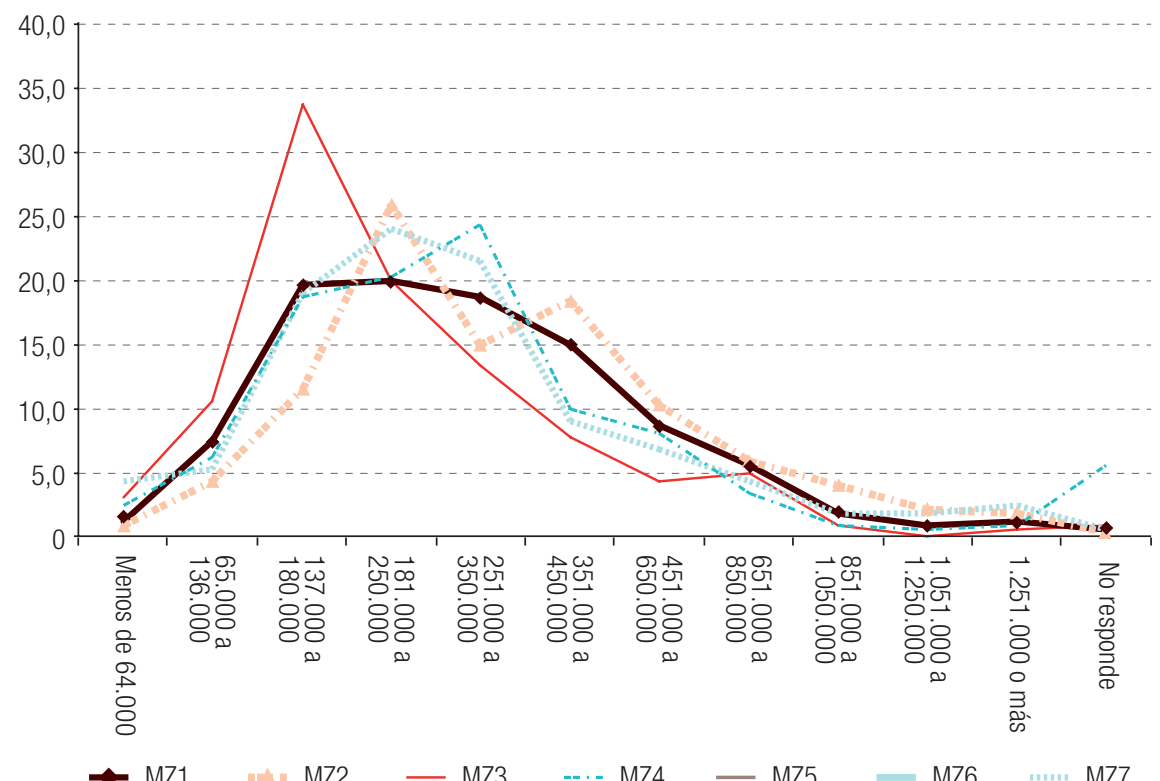

Fuente: Elaboración propia, sobre la base de datos de la Encuesta Nacional de Condiciones de Empleo, Trabajo, Salud y Calidad de Vida de los Trabajadores y Trabajadoras en Chile.

Lo interesante en este caso es la importancia que muestran las variables subjetivas. Los factores que más impacto tienen son si se siente bien o muy bien mentalmente o emocionalmente (BMENTAL2) y si disfruta con el trabajo que realiza (DISFTRAB2).

A su vez, la probabilidad de tener satisfacción laboral disminuye si las personas tienen siempre miedo al despido (MIEDODES) o si están desconformes con sus salarios (aquellos que indican que le gustaría que su salario aumente CONFSALA).

Otras variables como satisfacción con la promoción (SPROMO4), oportunidades de desarrollo (OPORTDESS), se siente razonablemente feliz (FELIZ2) y satisfacción con el ambiente laboral (SAMBIENT4) obtienen los resultados esperados. Se destaca que las dos primeras están asociadas a la administración empresarial, en cambio SAMBIENT4 se vincula con la organización de la empresa y FELIZ2 con factores subjetivos, emocionales. Las otras variables resultaron no tener impacto en la satisfacción laboral.

A nivel territorial se obtiene que el impacto de dos macrozonas (MZ2 y MZ7) desaparece. El resto se mantiene. 


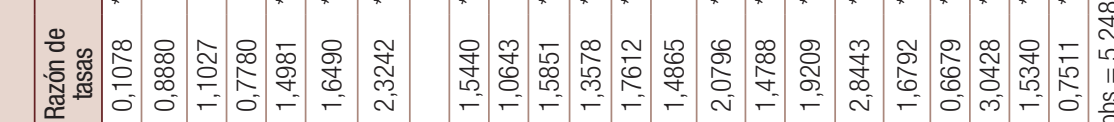

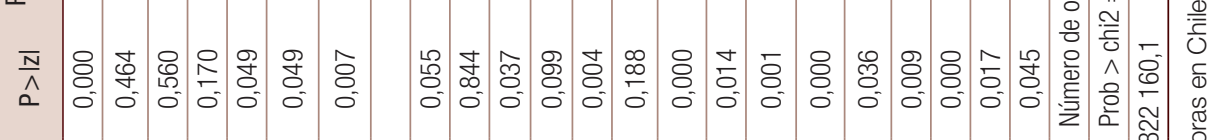

ن্ল

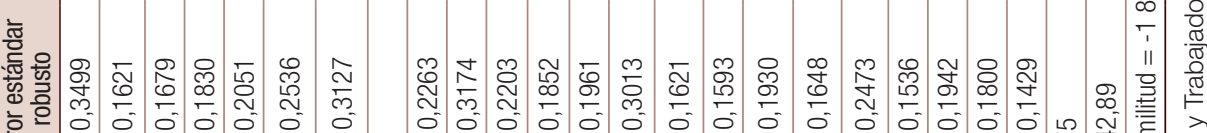
親

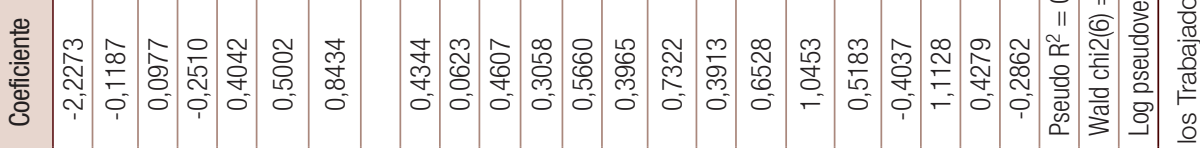

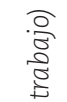

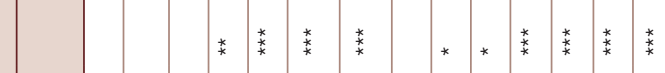

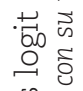

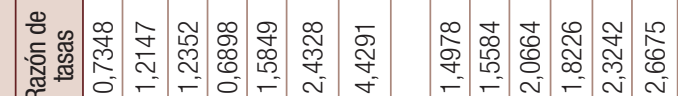

응

造

$m \stackrel{0}{0}$ है

ल

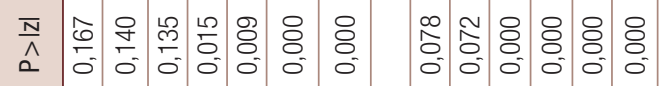

한

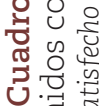

密율

స్లె

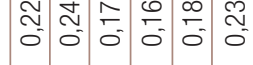

章

का के

के

근

幽 ह

อั

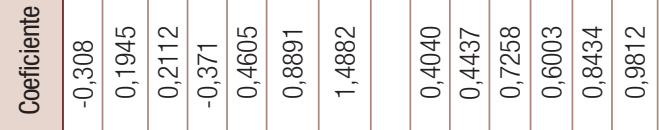

向

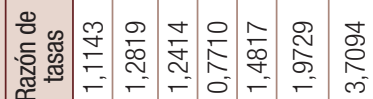

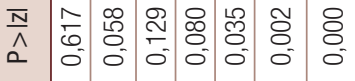

홓

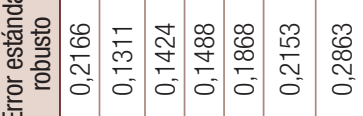

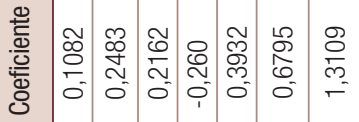

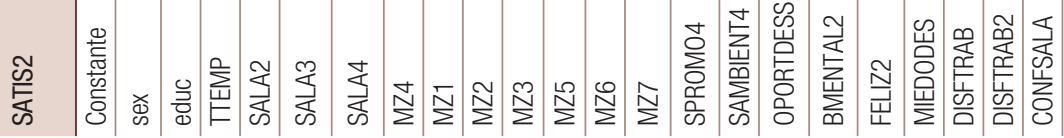

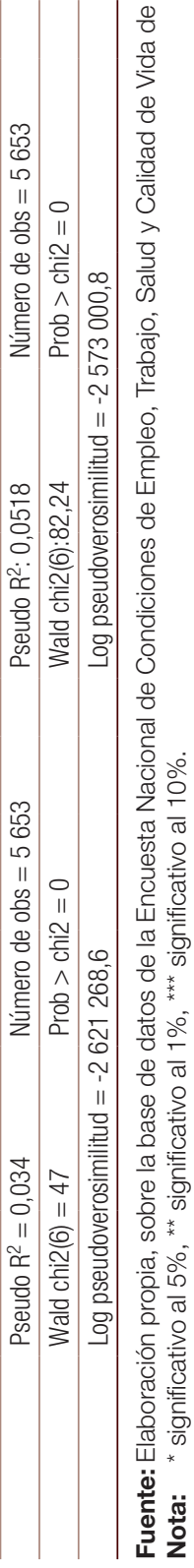




\section{Metodología de emparejamiento exacto}

El tema de interés ahora es poner a prueba los resultados anteriores en cuanto al impacto que tiene vivir en una macrozona distinta de la Región Metropolitana. Como se ha señalado en el apartado metodológico, para ello se aplica el emparejamiento, que es una técnica de análisis de impacto, en este caso el emparejamiento exacto.

La idea es realizar esta evaluación a partir de dos grupos de sujetos, con iguales características salvo que residen en zonas distintas. Para ello se debe realizar un emparejamiento, esto es, buscar un soporte común en ambos grupos. Las variables que se utilizan en este caso son aquellas que se incorporaron como determinantes en la estimación logit anterior (véase el cuadro $3 C$ ), tanto las variables objetivas como las subjetivas (sex, educ, TTEMP, SALA2, SALA3, SALA4, SPROMO4, SAMBIENT4, OPORTDESS, BMENTAL2, FELIZ2, MIEDODES, DISFTRAB, DISFTRAB2, CONFSALA).

Luego, se tiene una base emparejada en que los sujetos se diferencian solo por el lugar de residencia. De esta manera se estima la probabilidad de sentirse bien o muy bien con el trabajo, en un modelo logit con una sola variable explicativa, que identifica la macrozona en la que reside el individuo.

Se realizan comparaciones, primero entre los sujetos de la Región Metropolitana y el conjunto de las otras regiones del país, y luego entre la Región Metropolitana (MZ4) y cada una de las macrozonas, realizando dos tipos de estimaciones, con y sin la aplicación del método de emparejamiento exacto. Los resultados se presentan en el cuadro 4.

Como se observa, el indicador $\$ 1$ mejora sustancialmente en todos los casos, de este modo se concluye que el emparejamiento mediante la metodología de emparejamiento exacto permite obtener grupos comparables, mejorando el análisis. No obstante, se debe considerar que dicho procedimiento ha reducido entre un $54 \%$ y un $61 \%$ el número de observaciones.

Cuadro 4

Estimaciones con y sin la aplicación del método de emparejamiento exacto: impacto de la residencia en la probabilidad de satisfacción laboral

\begin{tabular}{|c|c|c|c|c|c|c|c|c|}
\hline & \multicolumn{7}{|c|}{ Impacto de las zonas de residencia en la probabilidad de satisfacción (zona base MZ4) } \\
\hline & & TODAS & MZ1 & MZ2 & MZ3 & MZ5 & MZ6 & MZ7 \\
\hline \multirow{7}{*}{$\begin{array}{l}\text { Antes del } \\
\text { emparejamiento }\end{array}$} & Estadístico $d 1$ & 0,60113 & 0,713060 & 0,710915 & 0,648887 & 0,670845 & 0,687866 & 0,717844 \\
\hline & $\mathrm{B}$ & $\begin{array}{l}0,4407^{\star \star *} \\
(0,1368)\end{array}$ & $\begin{array}{l}0,5780^{\star *} \\
(0,2497)\end{array}$ & $\begin{array}{l}0,5157^{\star \star} \\
(0,2431)\end{array}$ & $\begin{array}{l}0,5571^{\star * *} \\
(0,1917)\end{array}$ & $\begin{array}{c}0,2506 \\
(0,1596)\end{array}$ & $\begin{array}{l}0,5807^{\star \star \star} \\
(0,1820)\end{array}$ & $\begin{array}{l}0,9323^{\star \star *} \\
(0,2513)\end{array}$ \\
\hline & Constante & $\begin{array}{l}0,5351^{\star * *} \\
(0,0664)\end{array}$ & $\begin{array}{l}0,5351^{* * *} \\
(0,1197)\end{array}$ & $\begin{array}{l}0,5351^{\star * \star} \\
(0,1197)\end{array}$ & $\begin{array}{l}0,5351^{* * *} \\
(0,1197)\end{array}$ & $\begin{array}{l}0,5351^{* * *} \\
(0,1197)\end{array}$ & $\begin{array}{l}0,5351^{* * *} \\
(0,1197)\end{array}$ & $\begin{array}{l}0,5351^{* \star *} \\
(0,1197)\end{array}$ \\
\hline & Pseudo $\mathrm{R}^{2}$ & 0,0083 & 0,0029 & 0,0045 & 0,0096 & 0,0025 & 0,0089 & 0,0041 \\
\hline & Prob > chi2 & 0,0013 & 0,0029 & 0,0339 & 0,0037 & 0,1164 & 0,0014 & 0,0002 \\
\hline & Observ. SATIS2 = 0 & 1401 & 480 & 506 & 576 & 709 & 571 & 449 \\
\hline & Observ. SATIS = 1 & 3773 & 947 & 1125 & 1248 & 1417 & 1218 & 1028 \\
\hline \multirow{7}{*}{$\begin{array}{l}\text { Después de } \\
\text { aplicado el } \\
\text { emparejamiento }\end{array}$} & Estadístico $₫ 1$ & $1,913 e-15$ & $2,88 \mathrm{e}-16$ & $5,89 e-16$ & $3,29 e-16$ & $1,30 \mathrm{e}-15$ & $7,61 e-17$ & $7,42 \mathrm{e}-16$ \\
\hline & B & $\begin{array}{l}0,3902^{\star * *} \\
(0,1035)\end{array}$ & $\begin{array}{c}0,3003 \\
(0,2045)\end{array}$ & $\begin{array}{c}0,2952 \\
(, 2114)\end{array}$ & $\begin{array}{l}0,4196^{\star *} \\
(0,1713)\end{array}$ & $\begin{array}{l}0,3171^{\star *} \\
(0,1462)\end{array}$ & $\begin{array}{l}0,4751^{\star \star *} \\
(0,1705)\end{array}$ & $\begin{array}{l}0,8197^{\star \star \star} \\
(0,2695)\end{array}$ \\
\hline & Constante & $\begin{array}{l}1,1511^{* * *} \\
(0,057)\end{array}$ & $\begin{array}{c}1,010^{* \star *} \\
(0,1240)\end{array}$ & $\begin{array}{l}1,522^{\star \star *} \\
(0,1374)\end{array}$ & $\begin{array}{l}1,1984^{\star * *} \\
(0,1095)\end{array}$ & $\begin{array}{l}0,8739^{\star * *} \\
(0,1012)\end{array}$ & $\begin{array}{l}1,040^{\star \star *} \\
(0,1123)\end{array}$ & $\begin{array}{l}1,582^{\star \star *} \\
(0,1520)\end{array}$ \\
\hline & Pseudo $\mathrm{R}^{2}$ & 0,0052 & 0,0035 & 0,0033 & 0,0069 & 0,0042 & 0,0091 & 0,0225 \\
\hline & Prob > chi2 & 0,0001 & 0,1391 & 0,1606 & 0,0135 & 0,0298 & 0,0050 & 0,0016 \\
\hline & Observ. SATIS2 $=0$ & 737 & 332 & 360 & 468 & 470 & 411 & 306 \\
\hline & Observ. SATIS = 1 & 2143 & 226 & 322 & 417 & 502 & 411 & 265 \\
\hline
\end{tabular}

Fuente: Elaboración propia, sobre la base de datos de la Encuesta Nacional de Condiciones de Empleo, Trabajo, Salud y Calidad de Vida de los Trabajadores y Trabajadoras en Chile.

Nota: $\quad$ * significativo al $5 \%,{ }^{\star \star}$ significativo al $1 \%,{ }^{\star \star \star}$ significativo al 10\%, entre paréntesis desviaciones estándares robustas. 
Las estimaciones confirman el impacto a nivel global, es decir, vivir en una zona distinta de la Región Metropolitana impacta positivamente en la satisfacción laboral. Del análisis a nivel de macrozona se obtiene el mismo resultado para cuatro territorios (MZ3, MZ5, MZ6, MZ7); solo en las MZ1 y MZ2 no se observa un impacto significativo.

\section{Modelo multinomial}

En este punto se indaga en posibles diferencias en los impactos según el nivel de satisfacción, esto es, se calcula la probabilidad de sentirse bien o muy bien con su trabajo, en categorías diferentes, tal como se plantea en las ecuaciones (3) y (4).

Se estiman tres modelos, que se presentan en los cuadros 5A, 5B y 5C. En el primero, se aprecia que la propensión a estar muy satisfecho con el trabajo es mayor respecto de las otras opciones cuando hay un nivel educativo superior, mientras que el sexo deja de ser significativo. A su vez, el impacto de salarios más altos es mayor que en la opción 3 (se siente muy bien con el trabajo).

En el segundo modelo, al incorporar las variables de residencia (véase el cuadro 5B), se observan efectos similares a los mencionados en el modelo logit binario, aunque las zonas 1, 2 y 5 no son significativas.

En el cuadro 5C se expone el modelo con variables objetivas y de percepción, sin residencia. Lo interesante aquí es constatar la diferencia en el impacto que tienen las variables de percepción entre categorías. Por ejemplo, la satisfacción con el horario de trabajo (CHT) impacta positiva y significativamente en la posibilidad de sentirse muy bien con el trabajo, pero no en la categoría inferior (sentirse bien). A su vez, los sujetos con trabajo temporal (TTEMP) tienen una menor propensión a estar muy bien con su trabajo.

Por otro lado, se aprecia que algunos factores de percepción tienen efecto en ambas opciones pero con mayor impacto en la tercera, esto es, sentirse muy satisfecho. En este caso se encuentran las variables sentirse satisfecho con el ambiente de trabajo (SAMBIENT4), oportunidades de desarrollo (OPORTDESS) y siempre disfruta con el trabajo (DISFTRAB).

Sin embargo, las variables miedo al despido, conforme con el salario y sentirse feliz igual o más de lo habitual solo impactan en la propensión a estar satisfecho. Es posible que para estar muy satisfecho el nivel de exigencias sea mayor, lo que se evidencia en las variables DISFTRAB y DISFTRAB2. En este caso disfrutar siempre con el trabajo tiene una consecuencia favorable en las dos opciones, en cambio disfrutar siempre o casi siempre afecta positivamente solo en sentirse bien con el trabajo (la categoría 2). A su vez, entre quienes se sienten muy bien con el trabajo, en relación con las otras opciones, son 2,2 y 10,6 veces más cuando están bien y muy bien mental y emocionalmente (BMENTAL y BMENTAL2), respectivamente.

Este análisis revela la importancia que tienen en la satisfacción laboral variables de percepción asociadas a la gestión del recurso humano y al entorno organizacional. Los resultados son contundentes, los trabajadores lo valoran, inclusive más que elementos de condiciones laborales objetivas, lo que podría impactar tanto en la gestión organizacional como a nivel de política pública. 


\section{Cuadro 5}

Resultados obtenidos con los modelos multinomiales

(Opción base (=1) se siente menos que satisfecho con su trabajo)

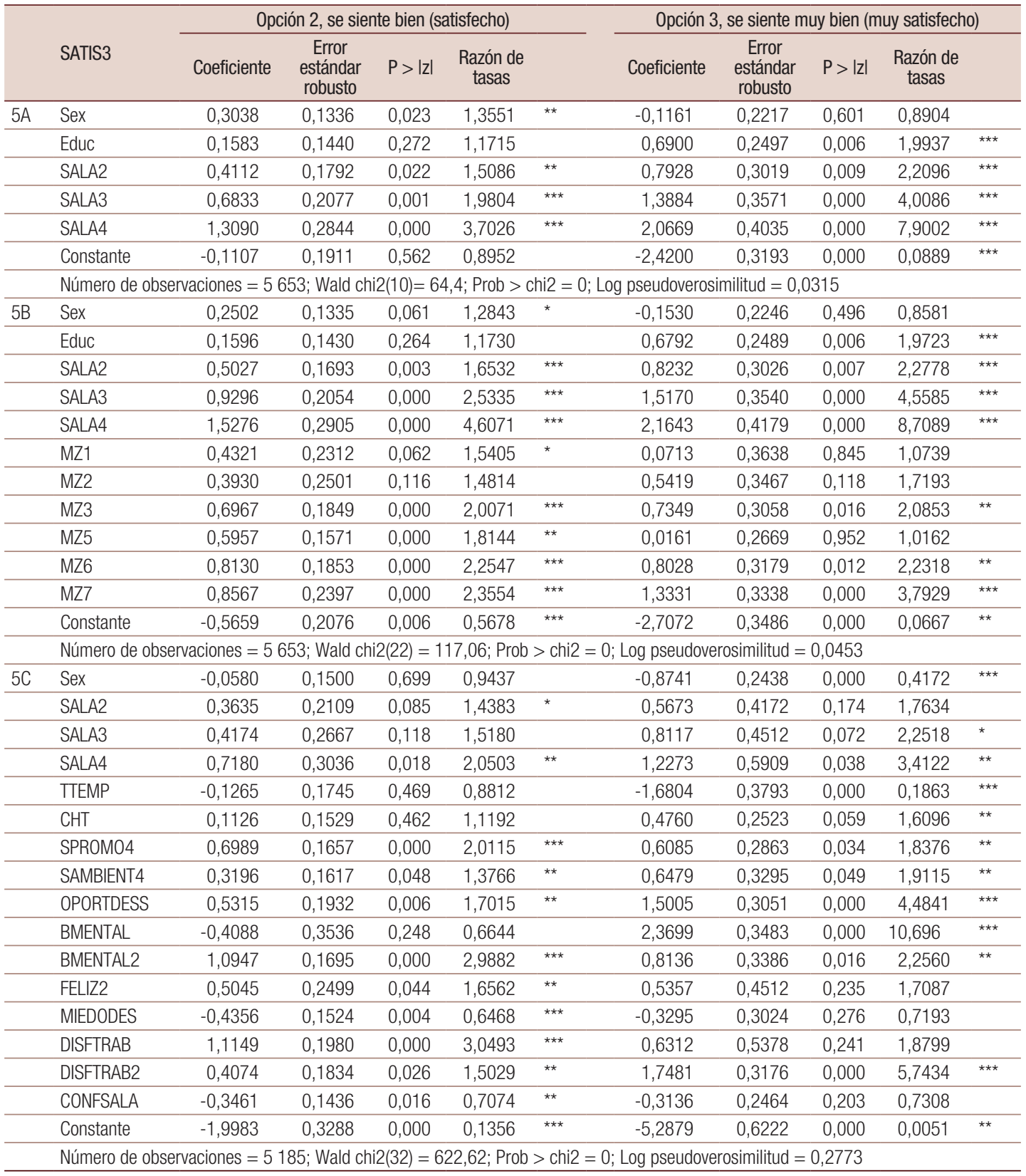

Fuente: Elaboración propia, sobre la base de datos de la Encuesta Nacional de Condiciones de Empleo, Trabajo, Salud y Calidad de Vida de los Trabajadores y Trabajadoras en Chile.

Nota: $\quad{ }^{\star}$ significativo al $5 \%,{ }^{\star \star}$ significativo al $1 \%,{ }^{* \star}$ significativo al $10 \%$.

\section{Conclusiones}

Conforme al propósito fijado inicialmente, se ha investigado la satisfacción laboral de trabajadores asalariados del sector privado en Chile, evaluado el impacto que tienen distintas variables y verificado diferencias en cuanto a la satisfacción que perciben los que viven en la Región Metropolitana y las 
otras zonas de Chile. Este trabajo se plantea como un aporte en la materia para el país, puesto que existe un déficit de estudios sobre el tema en que se considere el territorio.

La investigación se basa en los datos de la ENETS, realizada en el período 2009-2010, pues es la primera encuesta que incluye variables de percepción tan amplias y con representación regional.

Como se señaló, en la literatura internacional se presentan diversas conclusiones en cuanto al impacto del salario en la satisfacción laboral; los resultados de este trabajo indican que tiene un claro impacto positivo y significativo, lo que es consistente pues se obtiene que el efecto es mayor cuando el salario es también más alto y, cuando se analiza la variable dependiente en tres categorías, se obtiene un coeficiente mayor en la categoría 3, esto es, se siente muy bien con su trabajo (modelo multinomial).

Un aporte interesante de esta investigación son los resultados en cuanto a las variables de percepción. El impacto que se deriva de ellas es relevante, mayor que el de las objetivas. Es así como, del total de factores considerados inicialmente, los modelos finales (3C y $5 \mathrm{C}$ ) quedan conformados sobre todo con aspectos de percepción.

Las variables vinculadas a la calidad del empleo, como la seguridad social y el contrato, no son significativas; por otra parte, aun cuando el salario es la única variable objetiva que persiste, al incorporar los determinantes de percepción, estos son más significativos. Lo anterior es relevante, pues el grueso de la actuación de política pública en Chile se refiere a obtener condiciones mínimas en la calidad del empleo. Probablemente los trabajadores estén apreciando en mayor medida aspectos de percepción que se vinculan tanto a la gestión del recurso humano como a la organización.

Además, cuando se analiza la satisfacción laboral en tres categorías se obtienen diferencias notables en el aporte de las variables asociadas a la percepción.

No obstante lo anterior, la propensión a estar bien con el trabajo es mayor en las zonas distintas de la Región Metropolitana. Y cuando se analiza por zona, solo dejan de ser significativas las ubicadas en las macrozonas 1 y 2 , es decir, las ubicadas en el norte del país. Este hallazgo parece contradictorio por cuanto el salario en la Región Metropolitana es mayor, en términos relativos, además allí se debería concentrar una mayor cantidad de atractivos. En este trabajo no se han buscado las causas que podrían esclarecer esta situación, lo que, por supuesto, es un desafío que habrá que explorar. Por ahora, se exponen posibles explicaciones conforme a los resultados y con un cierto grado de intuición.

En primer lugar, haciendo una extrapolación de lo que se señala en la literatura, una hipótesis es que, dado que los salarios son menores en las otras regiones, particularmente las macrozonas 3, 4, 5 y 6 , tal vez en ellas se muestre una mayor satisfacción solo por el hecho de contar con empleo, en definitiva, es posible que el umbral para indicar satisfacción laboral sea menor.

Otro argumento es que, dado que las variables de percepción influyen mucho, es posible que junto a otras condicionantes asociadas al territorio (culturales, círculos sociales, familiares, entre otras), tengan un impacto mayor que el que tendría el salario, y ello podría a su vez explicar la baja movilidad laboral en Chile.

Por último, es posible que el salario tenga un comportamiento endógeno, provocado por una doble causalidad, es decir, la satisfacción laboral podría estar determinando el salario de los trabajadores; en este caso se confirmaría la importancia de los aspectos de percepción y, siguiendo con el argumento anterior, se explicaría el efecto positivo que tiene residir en una zona diferente de la Región Metropolitana, aunque es contradictorio con la alta concentración de la población en esta región.

Los resultados hasta ahora logrados, por un lado, revelan que es preciso abordar la temática a nivel territorial y reflejan la importancia de verificar posibles diferencias entre territorios, lo que tendría aplicación en la actuación de política pública, con acciones descentralizadas. Por otro lado, se obtiene información acerca del impacto que ejercen variables objetivas y de percepción, lo que resulta útil tanto en la generación de políticas públicas como en la administración de empresas, dado que señala 
la importancia que revisten en la satisfacción laboral variables asociadas a la gestión del recurso humano y al entorno organizacional. Una pregunta interesante aquí, que quedará abierta por ahora, es si la nueva gestión tiene efectos diferenciados según los territorios geográficos.

Finalmente, el trabajo apunta a la necesidad de contar con una versión más actualizada de encuestas que incluyan variables de percepción de la calidad del empleo, el desempeño, el entorno y la satisfacción laboral, a fin de verificar la consistencia temporal de los resultados.

\section{Bibliografía}

Aroca, P. y M. Atienza (2008), "La conmutación regional en Chile y su impacto en la Región de Antofagasta", EURE, vol. 34, N 102, Santiago, Pontificia Universidad Católica de Chile.

Aroca, P., J. D. Geoffrey y J. Paredes (2001), "Migracion interregional y el mercado laboral en Chile: 1977-82 y 1987-92", Cuadernos de Economía, vol. 38, №115, Santiago, Pontificia Universidad Católica de Chile.

Becker, G. (1964), Human Capital, Chicago, The University of Chicago Press.

Belfield, C. y R. Harris (2002), "How well do theories of job matching explain variations in job satisfaction across education levels? Evidence for UK graduates", Applied Economics, vol. 34, № 5, Taylor \& Francis.

Blackwell, M. y otros (2009), "CEM: coarsened exact matching in Stata", The Stata Journal, vol. 9, № 4.

Booth, A. y J. Van Ours (2007), "Job satisfaction and family happiness: the part-time work puzzle", Working Paper Series, № 100, Melbourne, Universidad de Melbourne.

BòriaReverter, S., M. CrespiVallbona y O. MascarillaMiró (2012), "Variables determinantes de la satisfacción laboral en España", Cuadernos de Economía, vol. 35, № 97, Elsevier.

Borra, C. y F. Gómez (2012), "Satisfacción laboral y salario: ¿compensa la renta laboral las condiciones no monetarias del trabajo?, Revista de Economía Aplicada, vol. 20, № 3, Universidad de Zaragoza.

Bryson, A., L. Cappellari y C. Lucifora (2004), "Does union membership really reduce job satisfaction?", British Journal of Industrial Relations, vol. 42, № 3, Wiley.

Card, D. y otros (2010), "Inequality at work: the effect of peer salaries on job satisfaction", NBER Working Paper, N ${ }^{\circ}$ 16396, Cambridge, Massachusetts, Oficina Nacional de Investigaciones Económicas.

Chiavenato, I. (2006), Introducción a la teoría general de la administración, McGraw-Hill.

Clark, A. (1997), "Job satisfaction and gender: why are women so happy at work?", Labour Economics, vol. 4, No 4, Amsterdam, Elsevier.

Clark, A. y A. Oswald (1996), "Satisfaction and comparison income", Journal of Public Economics, vol. 61, $N^{\circ} 3$, Amsterdam, Elsevier.

Clark, A. y F. Postel-Vinay (2009), "Job security and job protection", Oxford Economic Papers, vol. 61, № 2, Oxford University Press.

Clark, A., K. Nicolai y N. Westergård-Nielsen (2007), "Job satisfaction and co-worker wages: status or signal?", IZA Discussion Paper, № 3073.

De Vries, W. y otros (2015), "Conclusiones a contrapelo: la aportación de distintas carreras universitarias a la satisfacción en el empleo”, Revista de la Educación Superior, vol. 37, № 146, Asociación Nacional de Universidades e Instituciones de Educación Superior.

Farné, S. (2012), La calidad del empleo en América Latina a principios del siglo XXI, Bogotá, Universidad Externado de Colombia.

(2003), Estudio sobre la calidad de empleo en Colombia, Organización Internacional del Trabajo.

Farné, S. y A. Vergara (2007), "Calidad del empleo: ¿qué tan satisfechos están los colombianos con su trabajo?", Cuadernos de Trabajo, № 8, Bogotá, Universidad Externado de Colombia.

Ferrada, L. M. y P. Zarzosa (2010), "Diferencias regionales en la participación laboral femenina en Chile", Cuadernos de Economía, vol. 47, № 136, Santiago, Pontificia Universidad Católica de Chile.

Freeman, R. (1977), "Job satisfaction as an economic variable", NBER Working Paper, № 225, Cambridge, Massachusetts, Oficina Nacional de Investigaciones Económicas.

Gamero, C. (2003), "Análisis por género de la relación entre satisfacción laboral y tipo de contrato en España", Anales de Economía Aplicada 2007, Asociación Española de Economía Aplicada.

Greene, W. (1999), Análisis econométrico, Madrid, Prentice Hall.

Grund, C. y D. Sliwka (2007), "Reference-dependent preferences and the impact of wage increases on job satisfaction: theory and evidence", Journal of Institutional and Theoretical Economics (JITE), vol. 163, $N^{\circ}$ 2, Mohr Siebeck. 
Herzberg, F. (1962), "Basic needs and satisfactions of individuals", Industrial Relations Monograph, № 21. Herzberg, F., B. Mausner y B. Sryderman (1959), The Motivation to Work, Nueva York, John Wiley and Sons. lacus, S., G. King y G. Porro (2012), "Causal inference without balance checking: coarsened exact matching”, Political Analysis, vol. 20, № 1, Cambridge, Cambridge University Press.

Iglesias, C., R. Llorente y D. Dueñas (2010), "Calidad del empleo y satisfacción laboral", Investigaciones Regionales, № 19, Asociación Española de Ciencia Regional.

INE (Instituto Nacional de Estadísticas) (s/f) [en línea] http://www.ine.cl/canales/chile_estadistico/mercado_ del_trabajo/empleo/series_estadisticas/nuevas_empalmadas/series_fecha.php. (s/f) [en línea] http://www.ine.cl/canales/chile_estadistico/mercado_del_trabajo/remuneraciones/anuarios_ estadisticos/anuarios_estadisticos.php.

Jamett, I. y D. Paredes (2013), "Conmutación de larga distancia en Chile: estimando el premio por trabajar muy lejos de casa", Estudios de Economía, vol. 40, № 2, Santiago, Universidad de Chile.

Juárez-Adauta, S. (2012), "Clima organizacional y satisfacción laboral", Revista Médica del Instituto Mexicano del Seguro Social, vol. 50, № 3, Ciudad de México, Instituto Mexicano del Seguro Social.

Lévy-Garboua, L., C. Montmarquette y V. Simonnet (2007), "Job satisfaction and quits", Labour Economics, vol. 14, № 2, Amsterdam, Elsevier.

Long, J. y J. Freese (2014), Regression Models for Categorical Dependent Variables Using Stata, Stata Press.

Macedo, P. y R. Simões (1998), "Amenidades urbanas e correlação espacial: uma analise intra-urbana para BH (MG)", Revista Brasileira de Economia, vol. 4, № 52, Fundación Getulio Vargas.

McGregor, D. (1981), El aspecto humano de las empresas, Ciudad de México, Diana.

Mincer, J. (1958), "Investment in human capital and personal income distribution", Journal of Political Economy, vol. 66, $\mathrm{N}^{\circ} 4$, Chicago, The University of Chicago Press.

MINSAL (Ministerio de Salud) (2011), "Primera encuesta nacional de empleo, trabajo, salud y calidad de vida de los trabajadores y trabajadoras en chile (ENETS 2009-2010)" [en línea] http://epi.minsal.cl/ epi/Onotransmisibles/ENETS/INTERINSTITUCIONAL_BAJA.pdf.

(s/f), "Diseño conceptual y metodológico" [en línea] http://epi.minsal.cl/wp-content/uploads/2012/07/ Resumen_Ejecutivo_ENETS_Final.pdf.

Narbona, K. (2012), "La producción de compromiso en la empresa flexible", Santiago, Universidad de Chile [en línea] http://repositorio.uchile.cl/handle/2250/116785.

OIT (Organización Internacional del Trabajo) (1999), "Trabajo decente", Memoria del Director General, $87^{a}$ Reunión de la Conferencia Internacional del Trabajo, Ginebra.

Olarte, M. (2011), Los determinantes de la satisfacción laboral: una revisión teórica y empírica, Bogotá, Universidad Jorge Tadeo Lozano.

Pouliakas, K. e I. Theodossiou (2010), "Diferencias de satisfacción con el trabajo en Europa según nivel salarial", Revista Internacional del Trabajo, vol. 129, № 1, Organización Internacional del Trabajo.

Rocha, R. y A. Magalhães (2013), "Valoração das amenidades urbanas: uma estimação a partir dos diferenciais salariais e do custo de habitação para as regiões metropolitanas brasileiras", Revista de Economia Contemporanea, vol. 17, № 1, Río de Janeiro, Instituto de Economía de la Universidad Federal de Río de Janeiro.

Rodríguez-Gutiérrez, C. y J. Prieto-Rodríguez (2004), "Efecto de la afiliación sindical sobre la satisfacción laboral de los trabajadores en el caso español” [en línea] http://aeet-aede.ulpgc.es/Descargas/Sesion1Sala2/ Rodriguez-Prieto.pdf.

Sehnbruch, K. (2012), "La calidad del empleo en Chile: teoría y medición”, La calidad del empleo en América Latina a principios del siglo XXI, S. Farné (comp.), Bogotá, Universidad Externado de Colombia.

- (2008), "From the quantity to the quality of employment: an application of the capability approach to the Chilean labour market", The Capability Approach: Concepts, Measures and Applications, F. Comim, M. Qizilbash y S. Alkire, Nueva York, Cambridge University Press.

Sen, A. (2000), Desarrollo y libertad, Buenos Aires, Editorial Planeta.

Sloane, P. J. y H. Williams (2000), "Job satisfaction, comparison earnings, and gender", Labour, vol. 14, $N^{\circ} 3$, Wiley.

Somarriba, N. y otros (2010), "La calidad del trabajo en la Unión Europea", Estudios de Economía Aplicada, vol. $28, N^{\circ} 3$.

Stephen, R. y J. Timothy (2013), Comportamiento organizacional, Pearson. 